\title{
A retrospective population-based study of childhood hospital admissions with record linkage to a birth defects registry Lyn Colvin* and Carol Bower
}

\author{
Address: Telethon Institute for Child Health Research, Centre for Child Health Research, The University of Western Australia, Perth, WA, Australia \\ Email: Lyn Colvin* - lync@ichr.uwa.edu.au; Carol Bower - carolb@ichr.uwa.edu.au \\ * Corresponding author
}

Published: 10 May 2009

BMC Pediatrics 2009, 9:32 doi:10.1186/147|-2431-9-32

This article is available from: http://www.biomedcentral.com//47|-243|/9/32

(c) 2009 Colvin and Bower; licensee BioMed Central Ltd.

This is an Open Access article distributed under the terms of the Creative Commons Attribution License (http://creativecommons.org/licenses/by/2.0), which permits unrestricted use, distribution, and reproduction in any medium, provided the original work is properly cited.
Received: 26 November 2008

Accepted: 10 May 2009

\begin{abstract}
Background: Using population-based linked records of births, deaths, birth defects and hospital admissions for children born 1980-1999 enables profiles of hospital morbidity to be created for each child.

Methods: This is an analysis of a state-based registry of birth defects linked to population-based hospital admission data. Transfers and readmissions within one day could be taken into account and treated as one episode of care for the purposes of analyses ( $N=485,446$ children; 742,845 nonbirth admissions).

Results: Children born in Western Australia from 1980-1999 with a major birth defect comprised $4.6 \%$ of live births but $12.0 \%$ of non-birth hospital admissions from 1980-2000. On average, the children with a major birth defect remained in hospital longer than the children in the comparison group for the same diagnosis. The mean and median lengths of stay (LOS) for admissions before the age of 5 years have decreased for all children since 1980. However, the mean number of admissions per child admitted has remained constant at around 3.8 admissions for children with a major birth defect and 2.2 admissions for all other children.
\end{abstract}

Conclusion: To gain a true picture of the burden of hospital-based morbidity in childhood, admission records need to be linked for each child. We have been able to do this at a population level using birth defect cases ascertained by a birth defects registry. Our results showed a greater mean LOS and mean number of admissions per child admitted than previous studies. The results suggest there may be an opportunity for the children with a major birth defect to be monitored and seen earlier in the primary care setting for common childhood illnesses to avoid hospitalisation or reduce the LOS.

\section{Background}

Birth defects are a major source of infant and child morbidity and mortality. Each year in Western Australia (WA), birth defects (BDs) occur in 5\% of live births, more than $13 \%$ of stillbirths, $40 \%$ of neonatal deaths and $37 \%$ of post-neonatal deaths.[1] The burden of hospitalisation on these children is greater than for their peers, although most studies are either based around a single hospital setting and so not population-based; or, rely on morbidity codes indicating a BD diagnosis from the hospital records. [2-4] Australian statistics provide hospitalisation data based upon the principal diagnosis at discharge from hos- 
pital and for 1999-2000, "congenital anomalies" showed 35 admissions per 1,000 children up to the age of one year, accounting for $6 \%$ of all admissions in this age group. [5] These data are based upon hospital admissions that are not linked for individuals, and not linked to BD registries. As the data are only based on discharges where the diagnosis is coded as a congenital anomaly, they do not provide information on all hospital inpatient morbidity for the children with BDs nor how it is changing over time.

The aim of this study was to conduct a record linkage of population-based birth defects registry and hospital discharge data to demonstrate the true burden of hospitalisation for children with BDs and to compare admission rates and lengths of stay for common hospital admissions for children with and without a major birth defect.

\section{Methods}

This was a population-based historical cohort study of administrative data relating to all live births in WA from 1980 to 1999 inclusive $(\mathrm{N}=485,446)$ and hospital discharges from 1980-2000 inclusive. The WA Data Linkage System (WADLS) uses the Automatch software package with probabilistic matching based upon medical record number, surname, first given name, initial, date of birth, sex and address as the principal matching fields. Surnames are changed to a coded format in order to overcome the effects of most discrepancies in the spelling. First the NYSIIS (New York State Intelligence Information System) name compression algorithm is applied.[6] This carries out such tasks as bringing together commonly confused letter groups like 'ch' and 'gh' or 'sh' and 'sch' as well as removing vowels. The surnames are then Soundexed.[7], which involves giving the same code to similar sounding non-initial constants. The resulting compression and Soundex codes are assigned different weights for agreement depending upon their frequency in the population. Clerical checking of additional information is undertaken for possible matches that fall between definite matches and non-definite matches. Missed links have been estimated at $0.11 \%$. [8] According to Australian Census statistics, permanent migration out of WA in 2001 was $2.7 \%$ of the population.[9] The WADLS has been validated and has been used extensively for health research. [10-14] De-identified data were provided from the WADLS, linking the births and deaths data from the Midwives' Notification System and the Register of Births and Deaths, to the Birth Defects Registry, and to hospital admissions data from the Hospital Morbidity Data from 1980 to 2000 .

The Birth Defects Registry was established in 1980 and obtains high quality population-based information on BDs for all children born in WA.[1] Notifications to the
Registry are received from over 100 sources including paediatricians, obstetricians, cytogenetics and ultrasound departments, and all genetic counselling clinics. The Registry defines a BD as a structural or functional abnormality that is present at conception or occurs before the end of pregnancy, and is diagnosed by 6 years of age. Each defect (up to 10 per case) is coded according to the 5-digit British Paediatric Association ICD-9 system and is classified as major or minor according to a method devised by the Centers for Disease Control and Prevention. Most minor defects, unless disfiguring or requiring treatment, are not recorded in the Registry. Of all cases registered, about $90 \%$ have at least one major $\mathrm{BD}$. A list of exclusions can be found in the Registry's annual report.[1] Unless otherwise stated, results are for children with at least one major BD. Cases with more than one BD in a category were counted only once in each category. Those with BDs in more than one category were counted in each category. The comparison group was the population of children without a registered BD.

Comprehensive internal data validation was undertaken including the verification of date of birth, sex, birth outcome, and date and cause of death across the datasets used. The principal diagnosis for each admission to hospital is recorded on the hospital discharge summary form and is constructed from information available at the time of discharge from hospital. Discharges prior to July 1, 1999 were coded using the Ninth Revision of the International Classification of Diseases (ICD-9)[15] so discharges from July 1, 1999 to December 31, 2000 and coded using the Tenth Revision were mapped back to their equivalent ICD-9 code to allow comparisons across the admissions dataset. Validation of admission records included reviews of duplicate admission dates $(\mathrm{N}=1,401 /$ $1,007,780 ; 0.14 \%)$; an admission occurring before the last discharge from hospital ( $\mathrm{N}=519 ; 0.05 \%)$; and, re-admissions for the same event. Records were merged into one admission record when a child was readmitted within one day of a previous discharge for the same condition $(\mathrm{N}=$ 7,$467 ; 0.74 \%$ ). This mostly occurred when a child was transferred between hospitals. Where admission and discharge occurred on the same day, the LOS was considered 0.5 day, to reflect some period of hospitalization $(\mathrm{N}=$ $84,472 / 384,493 ; 22.0 \%$ ). Admissions associated with birth were excluded from all analyses. Seven admission age periods were used: up to 1 year, $>1-2$ years, $>2-3$ years, $>3-4$ years, $>4-5$ years, $>5-12$ years, and $>12-18$ years. Due to the cut-off point of 2000 for hospital admissions (dictated by the ethics approval for the study), complete years of follow up were not available for all children in all age periods; for example, admissions for a child born in 1993 would only be included up to their $5^{\text {th }}$ birthday in 1998 as they did not reach the age of 12 years by 2000 in order to be included in the 5-12 years age cohort. 
The denominators for the calculation of admissions per 1,000 children were the number of children alive at the beginning of the age period and the numerators were the number of children admitted. In order to adjust for the varying survival rates over the study period, results were stratified by age group and included only the children alive at the start of the age period. Child admission rates were calculated using rate ratios (RR), adjusting for year of birth. To assess the overall burden of hospitalization for children with a major BD up to the age of five years, specific BDs were analysed to find those with the highest number of admissions, the longest mean LOS for one admission, the greatest number of admissions per child, and the longest mean total LOS (summed over all admissions).

Data validation and analyses were undertaken using SAS version 9.1.[16] the SAS GENMOD procedure was used to calculate RR and 95\% confidence intervals; the SAS MEANS procedure to assess differences in means; and the NPAR1WAY procedure to assess differences in medians. The researchers received all data in a de-identified form from the WADLS. The person-based linkage was approved by the ethics and confidentiality committee of the Health Department of WA and permission to use the required data was obtained from the relevant data custodians.

\section{Results}

There were 25,734 live born children registered with a BD from 1980 to 1999 out of a total population of 485,446 live births (5.3\%). Of the children with BDs, $87.1 \%$ had at least one major $\mathrm{BD}$. Of the children with a major $\mathrm{BD}$, $10.5 \%(N=2,364)$ had more than two defects reported. Half of all children with a major BD belonged to only two diagnostic categories: $29.2 \%(\mathrm{~N}=6,546)$ with uro-genital defects only; $20.2 \%(\mathrm{~N}=4,516)$ with musculo-skeletal defects only (139 (0.6\%) children had defects in both categories). Excluding records of birth admissions and children with only a minor $\mathrm{BD}$, and after validation of the data, $49.5 \%(238,850 / 482,122)$ of all children had a record of at least one hospital admission from 1980 to 2000, with 616,976 hospital admission records available for analyses within the seven age periods. A flow chart of the study population selection is found in Figure 1.

\section{Hospital admissions of children with a major birth defect compared with all other live births}

Around half $(54.4 \%, \mathrm{~N}=250,219)$ of the children in the comparison group were admitted to hospital, compared to $83.2 \%(\mathrm{~N}=18,636)$ of the BDs group $(P<0.0001)$. Admissions for children with a BD accounted for $12.0 \%$ $(\mathrm{N}=89,008 / 742,845)$ of all admissions. In both groups, males were more likely to be admitted: $59.1 \%$ males and

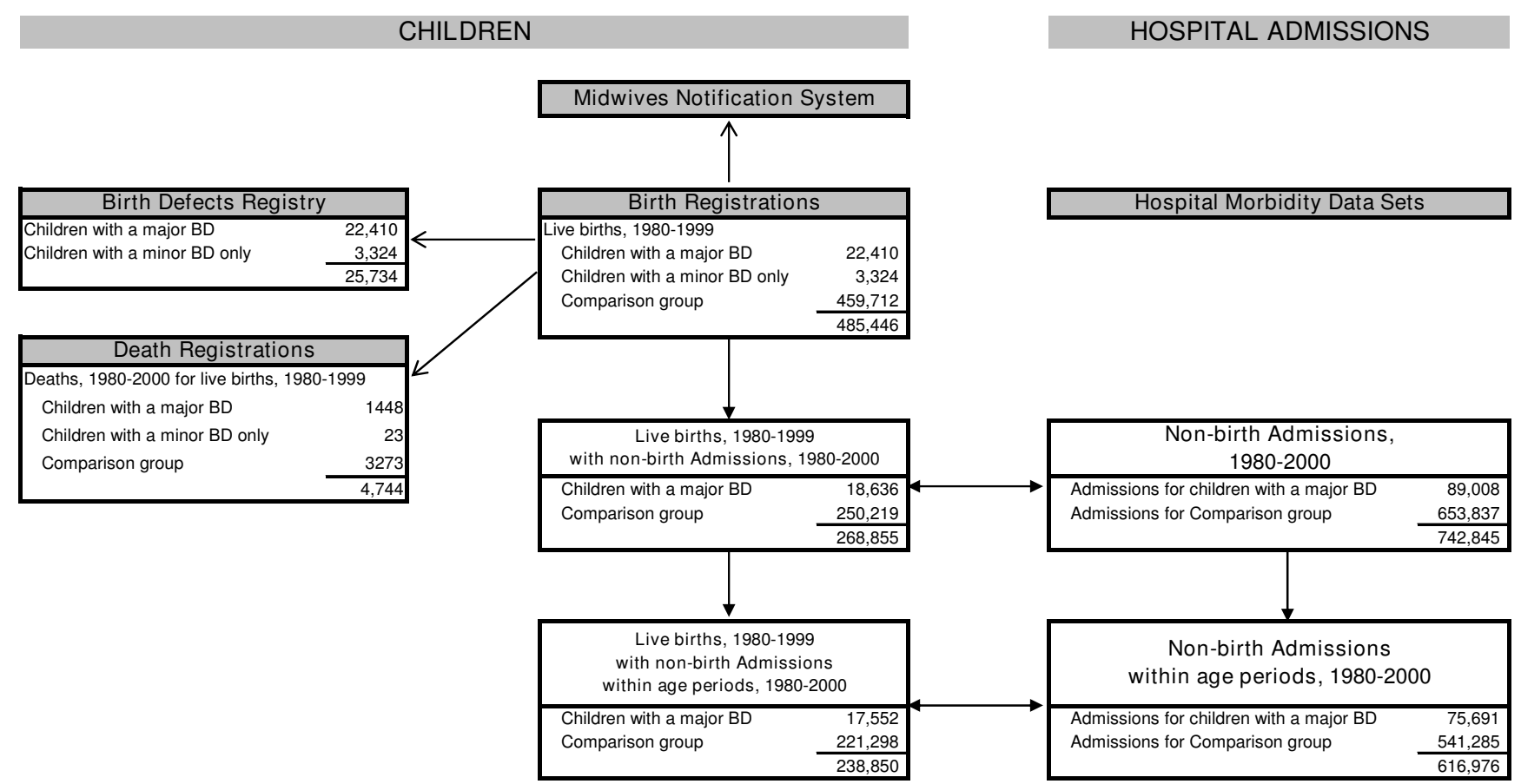

Figure I

Flow chart of data linkage for study population. 
$49.6 \%$ females were admitted within the comparison group; $88.8 \%$ males and $74.8 \%$ females within the BDs group $(P<0.0001)$. A child with a major BD was more likely to be admitted to hospital in all age periods and, in particular, excluding their birth admission, 2.5 times more likely to be admitted to hospital in each of the first 5 years of life than the comparison group (Table 1 ). In all age groups, the mean annual LOS for the BD group was highly significantly greater than the mean annual LOS for the comparison group $(P<0.0001)$. The median LOS for the $\mathrm{BD}$ group was also highly significantly greater than the median LOS for the comparison group up to 5 years of age (2.0 days vs. 1.0 days).

\section{Hospital admissions within major birth defect categories} In every age group, admission rates per 1,000 children alive at the start of the age period for every category of major BD were considerably higher than for the comparison children in the corresponding age group (Table 2). In all age groups, children in every BD category had longer mean LOS and mean annual LOS, compared with children without BDs (Table 3).

Leading causes of hospital admission up to 5 years of age The five most common causes for admission to hospital for the comparison group, for major BDs overall, and within each $\mathrm{BD}$ category were summarised using the first three digits of the ICD-9 code assigned as the principal diagnosis at hospital discharge (Table 4). Asthma (ICD-9 code 493) was the leading cause of admission in the comparison group and the second most common cause of admission for all major BDs. Asthma was one of the top five causes for admission in all but three of the $11 \mathrm{BD}$ categories (chromosome defects, nervous system defects and respiratory system defects). The mean LOS of 2.5 days for asthma admissions in the BDs group was significantly longer than the mean of 2.2 days for the comparison group: $P<0.0001$. Conditions related to $\mathrm{BDs}$ were the leading cause of admission in all categories of BDs.

The second most common reason for admission in the comparison group was for "780 general symptoms" and again the mean LOS was significantly longer for admissions for this reason in the BDs group: 3.7 days $\mathrm{cf}$. 2.0 days, $P<0.0001$. The mean LOS for the third most common reason for admission in the control group, "V65 other persons seeking consultation without complaint or sickness, " was not significantly different to the mean LOS for the BDs group. These children were predominantly from remote areas of the state. The mean LOS for the group of ICD-9 codes, "460-466 acute respiratory infections" was significantly longer in the BDs group than for the control group: 3.7 days cf. 2.8 days, $P<0.0001$.

\section{Changes in hospital admission rates up to 5 years of age}

Admissions per 1,000 live births decreased over time for the comparison group but increased in all BD categories. The mean total LOS per child up to the age of five years has decreased for most diagnostic categories of major BDs as well as the comparison group for admissions from 1980 to 1999 (Table 5). The number of admissions per child admitted increased for most BD categories and decreased for the comparison group. The mean LOS

Table I: Non-birth Admissions, births 1980-1999

\begin{tabular}{|c|c|c|c|c|c|c|c|c|c|}
\hline \multirow[b]{2}{*}{ Age Period } & \multicolumn{4}{|c|}{ Children with a Major Birth Defect } & \multicolumn{4}{|c|}{ Children without a Birth Defect (Comparison Group) } & \multirow[b]{2}{*}{$\begin{array}{l}\text { Rate Ratio } \\
\text { Children } \\
\text { Admitted } \\
(95 \% \mathrm{Cl})^{2}\end{array}$} \\
\hline & $\begin{array}{r}\text { No. } \\
\text { Children at } \\
\text { start of age } \\
\text { period }\end{array}$ & $\begin{array}{l}\text { \% Children } \\
\text { Admitted' }\end{array}$ & $\begin{array}{l}\text { Mean annual } \\
\text { LOS per } \\
\text { child } \\
\text { admitted } \\
\text { (days) }\end{array}$ & $\begin{array}{l}\text { Admissions } \\
\text { per Child } \\
\text { Admitted }\end{array}$ & $\begin{array}{r}\text { No. } \\
\text { Children at } \\
\text { start of age } \\
\text { period }\end{array}$ & $\begin{array}{l}\text { \% Children } \\
\text { Admitted }\end{array}$ & $\begin{array}{l}\text { Mean annual } \\
\text { LOS per } \\
\text { child } \\
\text { admitted } \\
\text { (days) }\end{array}$ & $\begin{array}{l}\text { Admissions } \\
\text { per Child } \\
\text { Admitted }\end{array}$ & \\
\hline$<=$ I year & 22,410 & 48.7 & 10.7 & 2.2 & 459,172 & 19.6 & 5.4 & 1.6 & $\begin{array}{c}2.48 \\
(2.45-2.52)\end{array}$ \\
\hline$>1-2$ years & 20,057 & 36.8 & 6.6 & 1.9 & 433,341 & 14.9 & 4.1 & 1.5 & $\begin{array}{c}2.46 \\
(2.42-2.5 \mathrm{I})\end{array}$ \\
\hline$>2-3$ years & 18,777 & 28.9 & 5.1 & 1.8 & 409,084 & 11.4 & 2.9 & 1.4 & $\begin{array}{c}2.53 \\
(2.47-2.59)\end{array}$ \\
\hline$>3-4$ years & 17,456 & 25.5 & 4.6 & 1.7 & 385,509 & 10.1 & 2.5 & 1.3 & $\begin{array}{c}2.52 \\
(2.45-2.59)\end{array}$ \\
\hline$>4-5$ years & 16,124 & 23.0 & 4.2 & 1.6 & 361,696 & 9.3 & 2.3 & 1.3 & $\begin{array}{c}2.46 \\
(2.39-2.54)\end{array}$ \\
\hline$>5-12$ years & 7,983 & 51.1 & 1.2 & 3.0 & 195,409 & 31.7 & 0.5 & 1.8 & $\begin{array}{c}1.61 \\
(1.58-1.65)\end{array}$ \\
\hline $\begin{array}{r}>12-18 \\
\text { years }\end{array}$ & 2,337 & 40.9 & 1.6 & 2.8 & 61,447 & 33.6 & 0.8 & 1.9 & $\begin{array}{c}1.22 \\
(1.16-1.28)\end{array}$ \\
\hline
\end{tabular}

\footnotetext{
I age group $>5-12$ yrs spans 7 years, $>12-18$ yrs spans 6 years. All other age groups included I year of follow up.
}

2 adjusted for year of birth 
Table 2: Number of children admitted per year, per 1000 children alive at the beginning of the age period, by diagnostic category and age period, births $1980-1999$

\begin{tabular}{|c|c|c|c|c|c|c|c|}
\hline \multirow[b]{2}{*}{ Diagnostic Category' } & \multicolumn{7}{|c|}{ Age Period } \\
\hline & $<=$ I year & $>1-2$ yrs & $>2-3$ yrs & $>3-4$ yrs & $>4-5$ yrs & $>5-12$ yrs & $>12-18 \mathrm{yrs}$ \\
\hline Cardiovascular Defects & $\mathrm{I}, 370$ & 860 & 592 & 495 & 424 & 232 & 147 \\
\hline Chromosome Defects & 1,282 & 1,252 & 919 & 703 & 780 & 411 & 256 \\
\hline CA of Ear, Face and Neck & 1,078 & 704 & 455 & 388 & 481 & 249 & 167 \\
\hline CA of Eye & I,802 & 1,165 & 875 & 700 & 641 & 345 & 203 \\
\hline $\mathrm{CA}$ of Integument & 962 & 652 & 437 & 437 & 416 & 282 & 193 \\
\hline Gastro-Intestinal Defects & 1,800 & 802 & 546 & 439 & 386 & 252 & 165 \\
\hline Musculo-Skeletal Defects & 830 & 555 & 385 & 323 & 300 & 184 & 174 \\
\hline Nervous System Defects & 1,474 & 1,363 & 993 & 880 & 818 & 516 & 399 \\
\hline Respiratory System Defects & $|, 23|$ & I,37| & 1,047 & 984 & 873 & 374 & 256 \\
\hline Uro-Genital Defects & 863 & 741 & 543 & 430 & 356 & 206 & 158 \\
\hline Other Major Defects² & 1,506 & $|| 4 \mid$, & 907 & 770 & 651 & 463 & 451 \\
\hline Any Major Birth Defect & 1,052 & 705 & 510 & 424 & 371 & 221 & 190 \\
\hline Comparison Group & 307 & 219 & 155 & 130 & 117 & 81 & 104 \\
\hline
\end{tabular}

I A child may be in more than one diagnostic category of major birth defects

2 These include: other and unspecified congenital anomalies, cystic fibrosis, hereditary haemolytic anaemias, congenital hypothyroidism, coagulation defects, neurofibromatosis, and muscular dystrophies and other myopathies

$\mathrm{CA}=$ congenital anomalies

decreased for all groups and the median LOS also decreased for most groups.

\section{Specific birth defects with the greatest burden of hospital} admission up to 5 years of age

Admissions up to 5 years of age were analysed for specific $\mathrm{BDs}$ (Table 6). The $\mathrm{BD}$ with the greatest proportion of overall admissions up to five years of age was undescended testis with $9.4 \%$ of all admissions $(15.3 \%$ of all male admissions). The greatest proportion of admissions for females was for developmental dysplasia of hip (9.7\%). The most number of admissions per child was for thalassaemias (50.5 admissions); 2.2 admissions for the comparison group. The longest mean LOS for a single

Table 3: Mean LOS for a single admission in days per child (and mean annual total LOS in days per child), by diagnostic category and age period, births $1980-1999$

Age Period

Diagnostic Category

Mean LOS (Mean annual LOS)

\begin{tabular}{|c|c|c|c|c|c|c|c|}
\hline & $<=$ I year & $>\mathrm{I}-2 \mathrm{yrs}$ & $>2-3$ yrs & $>3-4$ yrs & $>4-5$ yrs & $>5-12$ yrs & $>12-18 \mathrm{yrs}$ \\
\hline Cardiovascular Defects & $6.0(15.4)$ & $3.7(8.0)$ & $3.0(5.9)$ & $3.2(5.6)$ & $2.4(4.1)$ & $2.4(1.1)$ & $3.3(1.3)$ \\
\hline Chromosome Defects & $6.3(15.2)$ & $3.7(9.5)$ & $2.8(6.6)$ & $2.7(5.1)$ & $2.0(3.8)$ & $2.3(1.3)$ & $2.1(0.8)$ \\
\hline CA of Ear, Face and Neck & $4.8(10.6)$ & $4.0(7.1)$ & $1.8(2.9)$ & $1.9(3.1)$ & $2.8(4.5)$ & $2.5(1.1)$ & $3.1(1.1)$ \\
\hline CA of Eye & $4.8(13.6)$ & $2.6(5.7)$ & $2.4(4.9)$ & $2.1(3.7)$ & $2.5(4.2)$ & $2.5(1.3)$ & $2.7(1.2)$ \\
\hline $\mathrm{CA}$ of Integument & $4.0(8.0)$ & $2.1(4.3)$ & $1.8(2.9)$ & $1.6(2.7)$ & $2.0(3.1)$ & $1.8(1.0)$ & $4.7(2.0)$ \\
\hline Gastro-Intestinal Defects & $6.0(13.3)$ & $4.1(8.4)$ & $3.1(5.7)$ & $2.9(4.7)$ & $2.5(4.0)$ & $2.9(1.4)$ & $2.7(1.1)$ \\
\hline Musculo-Skeletal Defects & $4.9(10.7)$ & $3.7(7.7)$ & $3.3(5.9)$ & $2.9(4.9)$ & $2.9(4.9)$ & $3.0(1.3)$ & $4.0(1.7)$ \\
\hline Nervous System Defects & $6.7(19.4)$ & $4.8(12.7)$ & $4.6(10.5)$ & $4.2(8.5)$ & $4.1(8.9)$ & $4.0(3.0)$ & $5.0(3.8)$ \\
\hline Respiratory System Defects & $7.2(25.4)$ & $4.3(13.4)$ & $3.3(8.7)$ & $3.3(7.2)$ & $1.9(4.5)$ & $2.2(1.4)$ & $1.6(0.7)$ \\
\hline Uro-Genital Defects & $4.3(8.3)$ & $3.1(5.0)$ & $2.7(4.2)$ & $2.6(4.0)$ & $2.7(3.9)$ & $2.5(0.9)$ & $2.5(1.0)$ \\
\hline Other Major Defects² & $6.3(17.7)$ & $3.9(10.2)$ & $3.3(7.7)$ & $3.3(7.1)$ & $3.0(6.2)$ & $3.5(2.5)$ & $5.1(4.6)$ \\
\hline Any Major Birth Defect & $5.0(10.7)$ & $3.4(6.6)$ & $2.9(5.1)$ & $2.8(4.6)$ & $2.6(4.2)$ & $2.8(8.4)$ & $3.5(9.7)$ \\
\hline Comparison Group & $3.5(5.5)$ & $2.8(4.2)$ & $2.2(3.0)$ & $1.9(2.5)$ & $1.8(2.4)$ & $2.1(0.5)$ & $2.4(0.8)$ \\
\hline
\end{tabular}

I A child may be in more than one diagnostic category of major birth defects

2 These include: other and unspecified congenital anomalies, cystic fibrosis, hereditary haemolytic anaemias, congenital hypothyroidism, coagulation defects, neurofibromatosis, and muscular dystrophies and other myopathies

$\mathrm{CA}=$ congenital anomalies 
Table 4: Five leading causes of admission to hospital up to 5 years of age, by diagnostic category, births 1980-1995

\begin{tabular}{|c|c|c|c|c|c|c|c|c|}
\hline Diagnostic Category' & ICD-9 & Prinicipal Diagnosis & No. Adm & $(\%$ Adm) & No. Chn Adm & (\% Chn Adm) & $\mathrm{Adm} / \mathrm{Ch} \mathrm{Adm}$ & Mean LOS \\
\hline $\begin{array}{l}\text { Cardiovascular } \\
\text { Defects }\end{array}$ & 745 & $\begin{array}{l}\text { Bulbus cordis anomalies and } \\
\text { anomalies of cardiac septal } \\
\text { closure }\end{array}$ & 1,226 & $(13.2)$ & 635 & $(9.7)$ & 1.9 & 5.5 \\
\hline 9,259 admissions & 747 & $\begin{array}{l}\text { Other congenital anomalies of } \\
\text { circulatory system }\end{array}$ & 491 & $(5.3)$ & 363 & $(5.6)$ & 1.4 & 5.8 \\
\hline \multirow[t]{3}{*}{$\begin{array}{l}2,010 \text { children } \\
\text { admitted }\end{array}$} & 746 & $\begin{array}{l}\text { Other congenital anomalies of } \\
\text { heart }\end{array}$ & 408 & $(4.4)$ & 266 & $(4.1)$ & 1.5 & 5.4 \\
\hline & 465 & $\begin{array}{l}\text { Acute upper respiratory } \\
\text { infections of multiple or } \\
\text { unspecified sites }\end{array}$ & 368 & $(4.0)$ & 233 & (3.6) & 1.6 & 4.0 \\
\hline & 493 & Asthma & 367 & $(4.0)$ & 164 & $(2.5)$ & 2.2 & 2.8 \\
\hline $\begin{array}{l}\text { Chromosome } \\
\text { Defects }\end{array}$ & 381 & $\begin{array}{l}\text { Nonsuppurative otitis media and } \\
\text { Eustachian tube disorders }\end{array}$ & 213 & $(7.2)$ & 135 & $(6.7)$ & 1.6 & 0.6 \\
\hline 2,964 admissions & 745 & $\begin{array}{l}\text { Bulbus cordis anomalies and } \\
\text { anomalies of cardiac septal } \\
\text { closure }\end{array}$ & 156 & $(5.3)$ & 82 & $(4 . I)$ & 1.9 & 4.9 \\
\hline \multirow[t]{3}{*}{$\begin{array}{l}528 \text { children } \\
\text { admitted }\end{array}$} & 519 & $\begin{array}{l}\text { Other diseases of respiratory } \\
\text { system }\end{array}$ & 153 & $(5.2)$ & 72 & (3.6) & 2.1 & 5.8 \\
\hline & 465 & $\begin{array}{l}\text { Acute upper respiratory } \\
\text { infections of multiple or } \\
\text { unspecified sites }\end{array}$ & 146 & $(4.9)$ & 81 & $(4.0)$ & 1.8 & 4.5 \\
\hline & 464 & Acute laryngitis and tracheitis & 138 & $(4.7)$ & 81 & $(4.0)$ & 1.7 & 2.5 \\
\hline $\begin{array}{l}\text { CA of Ear, Face and } \\
\text { Neck }\end{array}$ & 744 & $\begin{array}{l}\text { Congenital anomalies of ear, face } \\
\text { and neck }\end{array}$ & 84 & $(16.8)$ & 72 & $(18.4)$ & 1.2 & 1.8 \\
\hline 500 admissions & 465 & $\begin{array}{l}\text { Acute upper respiratory } \\
\text { infections of multiple or } \\
\text { unspecified sites }\end{array}$ & 30 & $(6.0)$ & $\mathrm{II}$ & $(2.8)$ & 2.7 & 6.2 \\
\hline \multirow[t]{3}{*}{$\begin{array}{l}139 \text { children } \\
\text { admitted }\end{array}$} & 381 & $\begin{array}{l}\text { Nonsuppurative otitis media and } \\
\text { Eustachian tube disorders }\end{array}$ & 28 & $(5.6)$ & 23 & $(5.9)$ & 1.2 & 0.6 \\
\hline & 493 & Asthma & 15 & $(3.0)$ & 7 & $(1.8)$ & 2.1 & 5.4 \\
\hline & 550 & Inguinal hernia & 15 & $(3.0)$ & 13 & (3.3) & 1.2 & 2.7 \\
\hline CA of Eye & 743 & Congenital anomalies of eye & 496 & $(24.8)$ & 183 & $(14.5)$ & 2.7 & 2.3 \\
\hline 2,001 admissions & 381 & $\begin{array}{l}\text { Nonsuppurative otitis media and } \\
\text { Eustachian tube disorders }\end{array}$ & 79 & $(4.0)$ & 53 & $(4.2)$ & 1.5 & 0.8 \\
\hline \multirow{3}{*}{$\begin{array}{l}356 \text { children } \\
\text { admitted }\end{array}$} & 493 & Asthma & 61 & $(3.1)$ & 24 & $(1.9)$ & 2.5 & 2.8 \\
\hline & 465 & $\begin{array}{l}\text { Acute upper respiratory } \\
\text { infections of multiple or } \\
\text { unspecified sites }\end{array}$ & 57 & $(2.9)$ & 38 & $(3.0)$ & 1.5 & 5.9 \\
\hline & 783 & $\begin{array}{l}\text { Symptoms concerning nutrition, } \\
\text { metabolism and development }\end{array}$ & 47 & $(2.4)$ & 31 & $(2.5)$ & 1.5 & 6.4 \\
\hline CA of Integument & 228 & $\begin{array}{l}\text { Haemangioma and } \\
\text { lymphangioma, any site }\end{array}$ & 266 & $(13.8)$ & 188 & $(14.3)$ & 1.4 & 3.1 \\
\hline I,935 admissions & 757 & $\begin{array}{l}\text { Congenital anomalies of the } \\
\text { integument }\end{array}$ & 241 & $(12.5)$ & 83 & (6.3) & 2.9 & 0.8 \\
\hline \multirow{3}{*}{$\begin{array}{l}546 \text { children } \\
\text { admitted }\end{array}$} & 216 & Benign neoplasm of skin & 84 & (4.3) & 59 & $(4.5)$ & 1.4 & 3.3 \\
\hline & 493 & Asthma & 7I & (3.7) & 39 & $(3.0)$ & 1.8 & 2.2 \\
\hline & 780 & General symptoms & 65 & (3.4) & 43 & (3.3) & 1.5 & 2.1 \\
\hline $\begin{array}{l}\text { Gastro-Intestinal } \\
\text { Defects }\end{array}$ & 749 & Cleft palate and cleft lip & 922 & $(I I .2)$ & 557 & (9.3) & 1.7 & 8.8 \\
\hline 8,205 admissions & 750 & $\begin{array}{l}\text { Other congenital anomalies of } \\
\text { upper alimentary tract }\end{array}$ & 873 & $(10.6)$ & 766 & $(I 2.8)$ & I.I & 4.8 \\
\hline \multirow[t]{3}{*}{$\begin{array}{l}\text { I,947 children } \\
\text { admitted }\end{array}$} & 381 & $\begin{array}{l}\text { Nonsuppurative otitis media and } \\
\text { Eustachian tube disorders }\end{array}$ & 496 & $(6.1)$ & 342 & $(5.7)$ & 1.5 & 0.7 \\
\hline & 751 & $\begin{array}{l}\text { Other congenital anomalies of } \\
\text { digestive system }\end{array}$ & 439 & $(5.4)$ & 284 & $(4.7)$ & 1.5 & 9.3 \\
\hline & 493 & Asthma & 283 & $(3.5)$ & 123 & $(2.1)$ & 2.3 & 2.4 \\
\hline $\begin{array}{l}\text { Musculo-Skeletal } \\
\text { Defects }\end{array}$ & 754 & $\begin{array}{l}\text { Certain congenital } \\
\text { musculoskeletal deformities }\end{array}$ & 1,140 & $(I I .3)$ & 656 & $(9.1)$ & 1.7 & 3.6 \\
\hline 10,049 admissions & V54 & Other orthopaedic aftercare & 420 & $(4.2)$ & 270 & (3.8) & 1.6 & 1.4 \\
\hline $\begin{array}{l}2,610 \text { children } \\
\text { admitted }\end{array}$ & 493 & Asthma & 368 & (3.7) & 180 & $(2.5)$ & 2.0 & 2.7 \\
\hline
\end{tabular}


Table 4: Five leading causes of admission to hospital up to 5 years of age, by diagnostic category, births 1980-1995 (Continued)

\begin{tabular}{|c|c|c|c|c|c|c|c|c|}
\hline & 756 & $\begin{array}{l}\text { Other congenital musculoskeletal } \\
\text { anomalies }\end{array}$ & 352 & $(3.5)$ & 263 & (3.7) & 1.3 & 6.2 \\
\hline & 381 & $\begin{array}{l}\text { Nonsuppurative otitis media and } \\
\text { Eustachian tube disorders }\end{array}$ & 326 & (3.2) & 235 & (3.3) & 1.4 & 0.9 \\
\hline $\begin{array}{l}\text { Nervous System } \\
\text { Defects }\end{array}$ & 742 & $\begin{array}{l}\text { Other congenital anomalies of } \\
\text { nervous system }\end{array}$ & 311 & (6.3) & 190 & $(5.8)$ & 1.6 & 6.4 \\
\hline 4,968 admissions & 996 & $\begin{array}{l}\text { Complications peculiar to certain } \\
\text { specified procedures }\end{array}$ & 252 & $(5.1)$ & 115 & $(3.5)$ & 2.2 & 8.9 \\
\hline \multirow{3}{*}{$\begin{array}{l}786 \text { children } \\
\text { admitted }\end{array}$} & 780 & General symptoms & 227 & (4.6) & 139 & $(4.3)$ & 1.6 & 3.7 \\
\hline & 345 & Epilepsy & 220 & (4.4) & 70 & $(2.2)$ & 3.1 & 10.2 \\
\hline & 741 & Spina bifida & 168 & (3.4) & 102 & $(3.1)$ & 1.6 & 7.6 \\
\hline $\begin{array}{l}\text { Respiratory System } \\
\text { Defects }\end{array}$ & 748 & $\begin{array}{l}\text { Congenital anomalies of } \\
\text { respiratory system }\end{array}$ & 84 & $(10.0)$ & 45 & $(9.0)$ & 1.9 & 7.4 \\
\hline 838 admissions & 519 & $\begin{array}{l}\text { Other diseases of respiratory } \\
\text { system }\end{array}$ & 72 & $(8.6)$ & 25 & $(5.0)$ & 2.9 & 8.2 \\
\hline \multirow[t]{3}{*}{$\begin{array}{l}\text { II7 children } \\
\text { admitted }\end{array}$} & 465 & $\begin{array}{l}\text { Acute upper respiratory } \\
\text { infections of multiple or } \\
\text { unspecified sites }\end{array}$ & 40 & $(4.8)$ & 17 & (3.4) & 2.4 & 6.5 \\
\hline & 464 & Acute laryngitis and tracheitis & 34 & $(4 . I)$ & 16 & (3.2) & 2.1 & 1.5 \\
\hline & 381 & $\begin{array}{l}\text { Nonsuppurative otitis media and } \\
\text { Eustachian tube disorders }\end{array}$ & 34 & $(4 . I)$ & 19 & (3.8) & 1.8 & 1.2 \\
\hline Uro-Genital Defects & 752 & $\begin{array}{l}\text { Congenital anomalies of genital } \\
\text { organs }\end{array}$ & 3,524 & $(22.2)$ & 2,879 & $(23.3)$ & 1.2 & 2.5 \\
\hline 15,855 admissions & 599 & $\begin{array}{l}\text { Other disorders of urethra and } \\
\text { urinary tract }\end{array}$ & 675 & (4.3) & 449 & (3.6) & 1.5 & 4.4 \\
\hline \multirow[t]{3}{*}{$\begin{array}{l}4,959 \text { children } \\
\text { admitted }\end{array}$} & 593 & $\begin{array}{l}\text { Other disorders of kidney and } \\
\text { ureter }\end{array}$ & 671 & $(4.2)$ & 510 & $(4.1)$ & 1.3 & 5.6 \\
\hline & 493 & Asthma & 613 & (3.9) & 296 & (2.4) & 2.1 & 2.2 \\
\hline & 550 & Inguinal hernia & 457 & (2.9) & 422 & (3.4) & I.I & 1.6 \\
\hline Other Major Defects ${ }^{2}$ & 277 & $\begin{array}{l}\text { Other and unspecified disorders } \\
\text { of metabolism }\end{array}$ & 319 & (3.3) & 110 & $(1.9)$ & 2.9 & 11.4 \\
\hline 9,575 admissions & 519 & $\begin{array}{l}\text { Other diseases of respiratory } \\
\text { system }\end{array}$ & 313 & (3.3) & 157 & $(2.7)$ & 2.0 & 7.9 \\
\hline \multirow{3}{*}{$\begin{array}{l}\text { I,665 children } \\
\text { admitted }\end{array}$} & 493 & Asthma & 287 & (3.0) & 130 & $(2.2)$ & 2.2 & 3.1 \\
\hline & 780 & General symptoms & 281 & (2.9) & 186 & (3.2) & 1.5 & 3.5 \\
\hline & 783 & $\begin{array}{l}\text { Symptoms concerning nutrition, } \\
\text { metabolism and development }\end{array}$ & 274 & (2.9) & 185 & (3.2) & 1.5 & 6.2 \\
\hline $\begin{array}{l}\text { All Major Birth } \\
\text { Defects }\end{array}$ & 752 & $\begin{array}{l}\text { Congenital anomalies of genital } \\
\text { organs }\end{array}$ & 3,568 & (7.I) & 2,916 & (8.0) & 1.2 & 2.5 \\
\hline 50,432 admissions & 493 & Asthma & 1,903 & (3.8) & 883 & $(2.4)$ & 2.2 & 2.5 \\
\hline \multirow[t]{3}{*}{$\begin{array}{l}13,368 \text { children } \\
\text { admitted }\end{array}$} & 381 & $\begin{array}{l}\text { Nonsuppurative otitis media and } \\
\text { Eustachian tube disorders }\end{array}$ & $\mathrm{I}, 594$ & (3.2) & 1,152 & (3.2) & 1.4 & 0.8 \\
\hline & 465 & $\begin{array}{l}\text { Acute upper respiratory } \\
\text { infections of multiple or } \\
\text { unspecified sites }\end{array}$ & $\mathrm{I}, 457$ & (2.9) & 904 & $(2.5)$ & 1.6 & 2.6 \\
\hline & 780 & General symptoms & 1,278 & $(2.5)$ & 1,032 & $(2.8)$ & 1.2 & 3.7 \\
\hline Comparison Group & 493 & Asthma & 27,584 & (8.3) & 14,229 & $(5.1)$ & 1.9 & 2.2 \\
\hline 334,061 admissions & 780 & General symptoms & 16,930 & $(5.1)$ & 13,396 & $(4.8)$ & 1.3 & 2.0 \\
\hline \multirow[t]{3}{*}{$\begin{array}{l}\text { I54,5 I7 children } \\
\text { admitted }\end{array}$} & V65 & $\begin{array}{l}\text { Other persons seeking } \\
\text { consultation without complaint } \\
\text { or sickness }\end{array}$ & 13,930 & $(4.2)$ & 11,549 & $(4.1)$ & 1.2 & 3.3 \\
\hline & 466 & $\begin{array}{l}\text { Acute bronchitis and } \\
\text { bronchiolitis }\end{array}$ & 13,913 & $(4.2)$ & 11,058 & $(4.0)$ & 1.3 & 3.9 \\
\hline & 464 & Acute laryngitis and tracheitis & 12,192 & (3.6) & 10,064 & (3.6) & 1.2 & 1.8 \\
\hline
\end{tabular}

\footnotetext{
I A child may be in more than one diagnostic category of major birth defects

2 These include: other and unspecified congenital anomalies, cystic fibrosis, hereditary haemolytic anaemias, congenital hypothyroidism, coagulation defects, neurofibromatosis, and muscular dystrophies and other myopathies

$\mathrm{CA}=$ congenital anomalies
} 
Table 5: Change in admission rates up to 5 years of age, excluding birth admissions, births 1980-1994

\begin{tabular}{|c|c|c|c|c|c|c|c|}
\hline Birth Years & No. Chn Adm & No. Adm & Adm/I000 livebirths & Adm/Ch Adm & Mean LOS & Median LOS & $\begin{array}{c}\text { Mean Total LOS/Ch } \\
\text { Adm }\end{array}$ \\
\hline \multicolumn{8}{|l|}{$\begin{array}{l}\text { Cardiovascular } \\
\text { Defects }\end{array}$} \\
\hline $1980-1984$ & 528 & 2,412 & 3,327 & 4.6 & 5.1 & 2.0 & 23.2 \\
\hline $1985-1989$ & 626 & 2,763 & 3,313 & 4.4 & 4.9 & 2.0 & 21.6 \\
\hline $1990-1994$ & 721 & 3,524 & 3,589 & 4.9 & 4.2 & 2.0 & 20.4 \\
\hline \multicolumn{8}{|c|}{ Chromosome Defects } \\
\hline $1980-1984$ & 124 & 627 & 3,389 & 5.1 & 5.0 & 3.0 & 25.3 \\
\hline $1985-1989$ & 163 & 832 & 3,870 & 5.1 & 4.4 & 2.0 & 22.5 \\
\hline $1990-1994$ & 198 & 1,277 & 4,783 & 6.4 & 3.6 & 2.0 & 23.0 \\
\hline \multicolumn{8}{|c|}{$\begin{array}{l}\text { CA of Ear, Face and } \\
\text { Neck }\end{array}$} \\
\hline $1980-1984$ & 51 & 172 & 2,688 & 3.4 & 4.0 & 2.0 & 13.5 \\
\hline $1985-1989$ & 40 & 115 & 2,130 & 2.9 & 3.5 & 1.0 & 10.1 \\
\hline $1990-1994$ & 40 & 193 & 4,196 & 4.8 & 4.0 & 2.0 & 19.3 \\
\hline \multicolumn{8}{|l|}{ CA of Eye } \\
\hline $1980-1984$ & 92 & 483 & 4,025 & 5.3 & 3.8 & 2.0 & 20.0 \\
\hline $1985-1989$ & 97 & 528 & 4,632 & 5.4 & 3.3 & 1.0 & 18.1 \\
\hline $1990-1994$ & 149 & 917 & 5,660 & 6.2 & 3.3 & 1.0 & 20.1 \\
\hline \multicolumn{8}{|c|}{ CA of Integument } \\
\hline $1980-1984$ & 85 & 252 & 2,355 & 3.0 & 3.2 & 2.0 & 9.5 \\
\hline $1985-1989$ & 176 & 604 & 2,709 & 3.4 & 2.7 & 1.0 & 9.3 \\
\hline $1990-1994$ & 234 & 914 & 3,088 & 3.9 & 2.9 & 1.0 & 11.2 \\
\hline \multicolumn{8}{|l|}{$\begin{array}{l}\text { Gastro-Intestinal } \\
\text { Defects }\end{array}$} \\
\hline $1980-1984$ & 546 & 2,262 & 3,534 & 4.1 & 5.8 & 3.0 & 24.0 \\
\hline $1985-1989$ & 643 & 2,648 & 3,703 & 4.1 & 5.2 & 3.0 & 21.4 \\
\hline $1990-1994$ & 630 & 2,566 & 3,629 & 4.1 & 4.2 & 2.0 & I7.I \\
\hline \multicolumn{8}{|l|}{$\begin{array}{l}\text { Musculo-Skeletal } \\
\text { Defects }\end{array}$} \\
\hline $1980-1984$ & 748 & 2,749 & 2,061 & 3.7 & 5.1 & 2.0 & 18.7 \\
\hline $1985-1989$ & 794 & 3,160 & 2,367 & 4.0 & 4.2 & 2.0 & 16.7 \\
\hline $1990-1994$ & 878 & 3,431 & 2,329 & 3.9 & 3.6 & 1.0 & 14.1 \\
\hline \multicolumn{8}{|l|}{$\begin{array}{l}\text { Nervous System } \\
\text { Defects }\end{array}$} \\
\hline $1980-1984$ & 239 & $\mathrm{I}, 524$ & 4,293 & 6.4 & 7.2 & 3.0 & 45.9 \\
\hline $1985-1989$ & 230 & 1,386 & 3,971 & 6.0 & 5.3 & 2.0 & 31.9 \\
\hline $1990-1994$ & 281 & 1,848 & 4,981 & 6.6 & 4.3 & 2.0 & 28.3 \\
\hline \multicolumn{8}{|c|}{$\begin{array}{l}\text { Respiratory System } \\
\text { Defects }\end{array}$} \\
\hline $1980-1984$ & 28 & 221 & 2,483 & 7.9 & 8.0 & 3.0 & 63.1 \\
\hline $1985-1989$ & 36 & 200 & 2,128 & 5.6 & 4.4 & 1.5 & 24.4 \\
\hline $1990-1994$ & 44 & 385 & 4,583 & 8.8 & 4.3 & 2.0 & 37.6 \\
\hline \multicolumn{8}{|c|}{ Uro-Genital Defects } \\
\hline $1980-1984$ & 1,172 & 3,628 & 2,573 & 3.1 & 4.4 & 2.0 & 13.6 \\
\hline $1985-1989$ & 1,439 & 4,429 & 2,619 & 3.1 & 3.6 & 2.0 & 11.0 \\
\hline $1990-1994$ & 1,946 & 6,410 & 2,935 & 3.3 & 3.1 & 1.0 & 10.1 \\
\hline
\end{tabular}


Table 5: Change in admission rates up to 5 years of age, excluding birth admissions, births 1980-1994 (Continued)

\begin{tabular}{|c|c|c|c|c|c|c|c|}
\hline \multicolumn{8}{|c|}{ Other Major Defects } \\
\hline $1980-1984$ & 421 & 2,461 & 4,760 & 5.8 & 5.2 & 2.0 & 30.2 \\
\hline $1985-1989$ & 521 & 2,734 & 4,232 & 5.2 & 5.0 & 2.0 & 26.2 \\
\hline 1990-1994 & 597 & 3,582 & 4,776 & 6.0 & 4.1 & 1.0 & 24.5 \\
\hline \multicolumn{8}{|c|}{$\begin{array}{l}\text { All Major Birth } \\
\text { Defects }\end{array}$} \\
\hline 1980-1984 & 3,466 & 13,100 & 2,842 & 3.8 & 4.8 & 2.0 & 18.2 \\
\hline $1985-1989$ & 4,096 & 15,025 & 2,845 & 3.7 & 4.2 & 2.0 & 15.4 \\
\hline $1990-1994$ & 4,815 & 18,443 & 3,011 & 3.8 & 3.3 & 1.0 & 12.8 \\
\hline \multicolumn{8}{|c|}{ Comparison Group } \\
\hline $1980-1984$ & 45,514 & 103,785 & 977 & 2.3 & 3.4 & 2.0 & 7.8 \\
\hline $1985-1989$ & 48,209 & 103,764 & 892 & 2.2 & 2.8 & 2.0 & 6.1 \\
\hline $1990-1994$ & 50,244 & 104,836 & 874 & 2.1 & 2.3 & 1.0 & 4.9 \\
\hline
\end{tabular}

I These include: other and unspecified congenital anomalies, cystic fibrosis, hereditary haemolytic anaemias, congenital hypothyroidism, coagulation defects, neurofibromatosis, and muscular dystrophies and other myopathies

$\mathrm{CA}=$ congenital anomalies

admission was for cystic fibrosis (9.4 days); 2.8 days for the comparison group.

\section{Leading causes of admission within the comparison group up to 5 years of age (Table 7)}

Asthma and general symptoms were the top two reasons for admission in the comparison group up to 5 years. Children with a major BD were 1.3 times more likely to be admitted for asthma and 1.6 times more likely to be admitted for general symptoms. Children with a major BD had a mean total LOS in hospital that was one day longer than the children in the comparison group. For each of the leading 12 causes of admission within the comparison group, "V65 other persons seeking consultation without complaint or sickness" was the only one where the relative risk of admission for children with a BD was the same as for the comparison group. For all other diagnoses, children with a major BD had an increased risk of admission. The greatest RR of admission were admissions relating to 'nonsuppurative otitis media and eustachian tube disorders' $(\mathrm{RR}=2.4)$, 'other diseases of the respiratory system' $(R R=2.3)$, and 'acute upper respiratory infections of multiple or unspecified sites' $(\mathrm{RR}=2.0)$. The median LOS was similar for all children.

\section{Leading causes of admission for children aged 12 to 18 years}

Admissions for children in the older age periods were analysed. The RR of admission was still greater for the children with a major BD (40.9\% were admitted during the six year age period) compared with $33.6 \%$ of the comparison group $(R R=1.2)$. In this age group the leading cause for admission was for ICD-9 chapter 9, Diseases of the Digestive System (which includes 'diseases of oral cavity, salivary glands and jaws'), accounting for $19.0 \%$ of admissions in the comparison group and $14.8 \%$ in the major $\mathrm{BD}$ group. The next leading causes for admission in the comparison group were chapter 17 , Injury and Poisoning $(18.7 \%)$ and chapter 8, Diseases of the Respiratory System $(10.4 \%)$. For children with a major BD, the next leading causes for admission were chapter 3, Endocrine, Nutritional and Metabolic Diseases, and Immunity Disorders (14.0\%) and chapter 17, Injury and Poisoning (13.4\%).

\section{Discussion}

Our very large study of 485,446 live births provides population-based profiles of hospital admissions to enable comparisons between children with and without major BDs born over the same period in WA. Using linked administrative data for each live birth in WA from 19801999, hospital admissions for individual children were analysed within diagnostic categories of BDs, by age at admission and mean LOS. Net interstate migration from WA is low[9] and linkages with the deaths dataset meant the denominator data for specific age periods were more accurate. We found the admission rate up to the age of one year for a child with a major BD was 1,052 per 1,000 (1,108 per 1,000 males and 971 per 1,000 females) excluding the birth admission - three times the admission rate for children without a $\mathrm{BD}$. Whilst only $4.6 \%$ of live births in WA were children with a major $\mathrm{BD}$, this group accounted for $12.0 \%$ of the hospital admissions. This finding is similar to the almost $12 \%$ of paediatric hospitalisations related to birth defects and genetic diseases in California and South Carolina[4] but they are far higher than admissions for congenital anomalies (ICD-9 codes 740-759) alone in Australia ascertained by the national routinely collected statistics (35 per 1,000).[5] This occurs because, as we have shown, children with $\mathrm{BDs}$ are also more likely to be admitted for reasons other than a BD, and hospital discharge summaries may not always indicate that a child has a BD. Furthermore, there are also BDs classified with codes outside the ICD-9 chapter of 'Congenital Anomalies', such as thalassaemias and cystic fibro- 
Table 6: Specific birth defects with the greatest burden of hospital admission up to 5 years of age, excluding birth admissions, births $1980-1995$

\begin{tabular}{|c|c|c|c|}
\hline Greatest number of admissions & & & \\
\hline & Adm & Adm/Child Admitted & $\%$ Total Adm \\
\hline Undescended Testis & 6,249 & 2.8 & 9.4 \\
\hline Developmental Dysplasia of Hip & 3,476 & 3.0 & 5.3 \\
\hline Ventricular Septal Defect & 3,293 & 4.9 & 5.0 \\
\hline Vesico-Ureteric Reflux & 2,506 & 3.7 & 3.8 \\
\hline Talipes & 2,287 & 4.6 & 3.5 \\
\hline Pyloric Stenosis & 2,013 & 2.8 & 3.0 \\
\hline Down Syndrome & 1,924 & 5.6 & 2.9 \\
\hline Comparison Group & 334,061 & 2.2 & 100.0 \\
\hline
\end{tabular}

Most admissions per child

\begin{tabular}{|c|c|c|c|}
\hline & Adm & Adm/Child Admitted & Mean Total LOS \\
\hline Thalassemias & 202 & 50.5 & 29.8 \\
\hline Congenital Hydrocephalus (excludes those with NTD) & $\mathrm{I}, 007$ & 8.4 & 48.7 \\
\hline Tracheo-Oesophageal Fistula, Oesophageal Atresia/Stenosis & 636 & 8.0 & 41.3 \\
\hline Microcephaly & $\mathrm{I}, 05 \mathrm{I}$ & 7.8 & 62.5 \\
\hline Spina Bifida & 1,158 & 7.0 & 35.9 \\
\hline Hirschsprung's Disease & 468 & 6.9 & 49.7 \\
\hline Tetralogy of Fallot & 781 & 6.9 & 35.4 \\
\hline Comparison Group & 334,061 & 2.2 & 6.1 \\
\hline
\end{tabular}

Longest mean LOS (days) in one admission

\begin{tabular}{|c|c|c|c|}
\hline & Adm & Adm/Child Admitted & Mean LOS \\
\hline Cystic Fibrosis & 576 & 5.2 & 9.4 \\
\hline Cystic Kidney Disease & 270 & 4.7 & 8.0 \\
\hline Microcephaly & $|, 05|$ & 7.8 & 8.0 \\
\hline Hirschsprung's Disease & 468 & 6.9 & 7.2 \\
\hline Renal Agenesis or Dysgenesis & 248 & 6.5 & 6.6 \\
\hline Stenosis/Atresia Small Intestine & 270 & 4.7 & 6.3 \\
\hline Encephalocoele & 132 & 5.5 & 5.9 \\
\hline Comparison Group & 334,061 & 2.2 & 2.8 \\
\hline
\end{tabular}

Longest mean total LOS (days)

\begin{tabular}{|c|c|c|c|}
\hline & Adm & Adm/Child Admitted & Mean Total LOS \\
\hline Microcephaly & $\mid, 05 \mathrm{I}$ & 7.8 & 62.5 \\
\hline Hirschsprung's Disease & 468 & 6.9 & 49.7 \\
\hline Cystic Fibrosis & 576 & 5.2 & 49.2 \\
\hline Congenital Hydrocephalus (excludes those with NTD) & 1,007 & 8.4 & 48.7 \\
\hline Renal Agenesis or Dysgenesis & 248 & 6.5 & 43.2 \\
\hline Tracheo-Oesophageal Fistula, Oesophageal Atresia/Stenosis & 636 & 8.0 & 41.3 \\
\hline Cystic Kidney Disease & 270 & 4.7 & 38.0 \\
\hline Comparison Group & 334,061 & 2.2 & 6.1 \\
\hline
\end{tabular}

* A child may have more than one major birth defect 
Table 7: Leading causes of admission within the Comparison group compared with admissions for these reasons in children with a birth defect, up to 5 years of age, excluding birth admissions, births 1980-1995

\begin{tabular}{|c|c|c|c|c|c|c|c|c|c|c|}
\hline \multirow[b]{2}{*}{ ICD-9 } & \multirow[b]{2}{*}{$\begin{array}{l}\text { Principal } \\
\text { Diagnosis }\end{array}$} & \multicolumn{2}{|c|}{$\begin{array}{c}\text { Adm } \\
\mathrm{N}=384,494\end{array}$} & \multicolumn{2}{|c|}{$\begin{array}{l}\text { Children Admitted } \\
\qquad N=167,885\end{array}$} & \multirow{2}{*}{$\begin{array}{c}\begin{array}{c}\text { Rate Ratio } \\
\text { Children }\end{array} \\
\begin{array}{c}\text { Admitted } \\
(95 \% \mathrm{Cl})^{\prime}\end{array} \\
\begin{array}{c}\text { Major } \\
\text { BD }\end{array}\end{array}$} & \multicolumn{2}{|c|}{$\begin{array}{l}\text { Mean Total LOS } \\
\text { (days) }\end{array}$} & \multicolumn{2}{|c|}{$\begin{array}{l}\text { Median LOS } \\
\quad \text { (days) }\end{array}$} \\
\hline & & $\begin{array}{l}\text { Major BD } \\
N=50,432\end{array}$ & $\begin{array}{l}\text { Comparison } \\
N=334,061\end{array}$ & $\begin{array}{l}\text { Major BD } \\
N=13,368\end{array}$ & $\begin{array}{l}\text { Comparison } \\
N=154,517\end{array}$ & & $\begin{array}{l}\text { Major } \\
\text { BD }\end{array}$ & Comparison & $\begin{array}{l}\text { Major } \\
\text { BD }\end{array}$ & Comparison \\
\hline 493 & Asthma & 1,903 & 27,584 & 883 & 14,229 & $\begin{array}{c}1.30 \\
(1.22-1.39)\end{array}$ & 5.5 & 4.3 & 2.0 & 2.0 \\
\hline 780 & $\begin{array}{l}\text { General } \\
\text { symptoms }\end{array}$ & I,457 & 16,930 & 1,032 & 13,396 & $\begin{array}{c}1.63 \\
(1.54-1.74)\end{array}$ & 3.7 & 2.6 & 2.0 & 1.0 \\
\hline V65 & $\begin{array}{l}\text { Other persons } \\
\text { seeking } \\
\text { consultation } \\
\text { without } \\
\text { complaint or } \\
\text { sickness }\end{array}$ & 683 & 13,930 & 546 & 11,549 & $\begin{array}{c}1.01 \\
(0.93-1.10)\end{array}$ & 3.8 & 4.0 & 2.0 & 2.0 \\
\hline 466 & $\begin{array}{l}\text { Acute bronchitis } \\
\text { and bronchiolitis }\end{array}$ & 1,229 & 13,913 & 942 & 11,058 & $\begin{array}{c}1.78 \\
(1.67-1.90)\end{array}$ & 6.8 & 4.9 & 4.0 & 3.0 \\
\hline 464 & $\begin{array}{l}\text { Acute laryngitis } \\
\text { and tracheitis }\end{array}$ & 906 & 12,192 & 643 & 10,064 & $\begin{array}{c}1.36 \\
(1.26-1.47)\end{array}$ & 2.9 & 2.2 & 1.0 & 1.0 \\
\hline 009 & $\begin{array}{l}\text { Ill-defined } \\
\text { intestinal } \\
\text { infections }\end{array}$ & 875 & 12,165 & 652 & 9,476 & $\begin{array}{c}1.50 \\
(1.39-1.62)\end{array}$ & 5.6 & 5.1 & 2.0 & 2.0 \\
\hline 465 & $\begin{array}{l}\text { Acute upper } \\
\text { respiratory } \\
\text { infections of } \\
\text { multiple or } \\
\text { unspecified sites }\end{array}$ & 1,278 & 11,994 & 904 & 9,716 & $\begin{array}{c}1.98 \\
(1.86-2.12)\end{array}$ & 5.2 & 3.4 & 2.0 & 2.0 \\
\hline 381 & $\begin{array}{l}\text { Nonsuppurative } \\
\text { otitis media and } \\
\text { Eustachian tube } \\
\text { disorders }\end{array}$ & 1,594 & 11,723 & 1,152 & 9,733 & $\begin{array}{c}2.40 \\
(2.26-2.55)\end{array}$ & 1.1 & 0.8 & 0.5 & 0.5 \\
\hline 474 & $\begin{array}{l}\text { Chronic disease } \\
\text { of tonsils and } \\
\text { adenoids }\end{array}$ & 701 & 10,889 & 671 & 10,493 & $\begin{array}{c}1.33 \\
(1.24-1.44)\end{array}$ & 1.7 & 1.6 & 1.0 & 1.0 \\
\hline 519 & $\begin{array}{l}\text { Other diseases } \\
\text { of respiratory } \\
\text { system }\end{array}$ & 1,188 & 8,477 & 671 & 6,202 & $\begin{array}{c}2.31 \\
(2.14-2.50)\end{array}$ & 9.9 & 5.3 & 3.0 & 3.0 \\
\hline 382 & $\begin{array}{l}\text { Suppurative and } \\
\text { unspecified otitis } \\
\text { media }\end{array}$ & 693 & 8,262 & 568 & 7,069 & $\begin{array}{c}1.68 \\
(1.54-1.83)\end{array}$ & 2.7 & 2.4 & 1.0 & 1.0 \\
\hline 521 & $\begin{array}{l}\text { Diseases of hard } \\
\text { tissues of teeth }\end{array}$ & 713 & 8,011 & 651 & 7,507 & $\begin{array}{c}1.80 \\
(1.67-1.95)\end{array}$ & 0.8 & 0.7 & 0.5 & 0.5 \\
\hline
\end{tabular}

I adjusted for year of birth

sis and these conditions can have substantial hospital morbidity. An analogous situation was observed in a study of mortality associated with birth defects[17], which demonstrated that using birth defects registry data gave a more comprehensive picture of the full burden of birth defects on mortality than was evident from cause of death statistics alone.

By excluding birth admissions, issues relating to well-baby admissions and birth circumstances can be separated from the ongoing burden of hospitalisation a child may experience. This was highlighted in the report by MacFaul and Werneke showing that large numbers of wellborn infants artificially inflate the paediatric hospital admis- sion statistics.[18] However, to the extent that prolonged birth admissions in children with BDs and other serious perinatal conditions may occur, our data will underestimate the total burden.

Whilst admissions per 1000 children alive at the start of the age period and admissions per child admitted have decreased in the comparison group, these rates have generally increased within most categories of BD. There are several possible reasons for these trends. Many procedures are now done on an outpatient basis that formerly required hospital admission and this change may be more likely to occur for a child without a BD. New investigations and new treatment methods may require hospital 
admission in some children with BDs, and improved survival of children with BDs may result in increased hospitalisation. We have seen an example of this in WA in children with Down syndrome. There has been a significant increase in overall survival in these children since 1980 and a significant increase in survival for children with congenital heart disease and Down syndrome, who are very likely to require several hospital admissions.[19] There may also be an increasing tendency to admit children with BDs to hospital for preventive procedures or medication stabilization (eg changing anticonvulsant drugs, dental procedures[20]).

Mean and median LOS have decreased over time in both groups. This is probably due to the fact that many more procedures that once required a longer admission to hospital are now done as a day admission. Our analyses showed a greater mean LOS and mean number of admissions per child admitted than previous studies for children with a major BD. These children were admitted to hospital for longer even when admitted for non-BD conditions. For the most common reasons for admission in the comparison group up to 5 years of age, asthma and general symptoms, children with a $\mathrm{BD}$ were $30 \%$ more likely to be admitted for asthma and 60\% more likely to be admitted for general symptoms. These children also remained in hospital longer than the children in the comparison group, but the median LOS was similar in both groups, indicating that some admissions in the major BD group had extended LOS. Possible reasons for this include greater complexity in treating children with BDs and identification of co-morbidities whilst in hospital that are then treated and thus delay discharge. Unfortunately, our dataset did not contain information with which we could address the potential reasons for longer stays and more admissions over time.

A 2004 report reviewed the records of 5,747 consecutive admissions to a paediatric hospital during 1996 for 4,224 children and found $71 \%$ of these children had a condition with a 'significant genetic component'.[3] Children with single-gene and chromosomal disorders had an average hospital stay (7.1 days) that was twice as long as the 3.5 days average stay for children without any pre-existing chronic medical disorder. Other similar studies have also been reported $[3,4,21,22]$ but all of these relied upon the hospital diagnoses data for ascertaining cases of BDs and were likely to underestimate the true hospital morbidity rate per child.

The previous literature suggests that children with BDs have increased rates of hospital admission and longer stays in hospital but because of limitations in case ascertainment these may still be underestimated.
We found similar results to Viner[23] in the 12-18 years age group where females surpassed males in admissions per 1000 alive at 12 years, admissions per child admitted, and in mean LOS. In all other age groups, males ranked above females for these three measures, regardless of whether the child had a birth defect or not.

Newacheck et al. reported information on the extent to which out-of-pocket expenses are financially burdensome for families of children with special health care needs and found these expenses to be three times higher when compared with other children.[24] Our study provides more details of the burden on families of children with a $\mathrm{BD}$ with regard to hospital admissions - these children will attend hospital more often and have longer LOS, than for other children of their own age even for admissions not directly due to their BD. This was particularly evident for the children in the younger ages. Waitzman et al.[25] estimated the economic costs of 18 of the most clinically significant birth defects using the California Birth Defects Monitoring Program. However, as the children were not linked to their hospital admissions the study needed to make allowances for "missing discharges" - admissions where their condition might be considered incidental to the primary reason for admission. Our study was able to detect all such admissions for all children registered with a birth defect in WA.

Children with a major BD were 2.4 times more likely to be admitted for nonsuppurative otitis media. Casselbrant et al. outlined various risk factors considered important in the occurrence, recurrence, and persistence of middle-ear disease, including immunocompetence (as in Down syndrome) and craniofacial abnormalities such as cleft palate.[26] Children with Down syndrome also have abnormalities of their Eustachian tube, predisposing them to middle ear infections.[27]

\section{Conclusion}

Whilst it is encouraging that the mean and median LOS in hospital for admissions before the age of 5 years has decreased for all children since 1980, the mean number of admissions per child admitted has remained constant at around 2.2 admissions for children without BDs and 3.8 admissions for children with a BD. Our linked data could be used to investigate further the public health impact of medical care utilisation for children with BDs in Australia, as has been called for in the US.[28] Collection of additional data could assist in ascertaining the reasons for the increase in admissions in children with BDs and whether outcomes have improved. These findings also raise the question of whether there is an opportunity for children with BDs to be monitored and seen earlier in the primary care setting for childhood illnesses not associated with 
treatment of the $\mathrm{BD}$ - to avoid hospitalisation or reduce the LOS.

\section{Competing interests}

The authors declare that they have no competing interests.

\section{Authors' contributions}

Both authors 1) have made substantial contributions to conception and design, or acquisition of data, or analysis and interpretation of data; 2) have been involved in drafting the manuscript or revising it critically for important intellectual content; and 3) have given final approval of the version to be published.

\section{Authors' information}

Clinical Professor Carol Bower is the Medical Specialist for the Western Australian Birth Defects Registry.

\section{Acknowledgements}

Our thanks to colleagues at the WA Birth Defects Registry and the Telethon Institute for Child Health Research who supported this study and, in particular, to Peter Cosgrove for assistance with data extraction from the WADLS. The study was supported by funding from National Health and Medical Research Council Program Grants (003209, 3535I4); an Australian Postgraduate Award scholarship (to LC); and, National Health and Medical Research Council Fellowships (I72303, 353628 to CB). The funding bodies did not have any role in the study design or conduct, manuscript preparation or submission.

\section{References}

I. Bower C, Rudy E, Callaghan A, Cosgrove P, Quick J: Report of the Birth Defects Registry of Western Australia, 1980-2006. 2007 [http://www.kemh.health.wa.gov.au/services/birth defects/ index.htm]. Subiaco: King Edward Memorial Hospital, Women and Newborn Health Service Report No.: 14

2. Billett J, Majeed A, Gatzoulis M, Cowie M: Trends in hospital admissions, in-hospital case fatality and population mortality from congenital heart disease in England, 1994 to 2004. Heart 2008, 94:342-348.

3. McCandless SE, Brunger JW, Cassidy SB: The burden of genetic disease on inpatient care in a children's hospital. Am J Hum Genet 2004, 74:121-127.

4. Yoon PW, Olney RS, Khoury MJ, Sappenfield WM, Chavez GF, Taylor $D$ : Contribution of birth defects and genetic diseases to pediatric hospitalizations: a population-based study. Arch Pediatr Adolesc Med 1997, I 5 I: 1096-I I03.

5. Al-Yaman F, Bryant M, Sargeant H: Australia's children: their health and wellbeing 2002. AlHW Cat. No. PHE 36 Canberra: AlHW; 2002.

6. Newcombe HB: Handbook of Record Linkage, Appendix H New York: OUP; 1988.

7. Knuth DE: The Art of Computer Programming. Sorting and Searching Volume 3. Reading, MA: Addison-Wesley; 1973.

8. Holman CD, Bass AJ, Rouse IL, Hobbs MS: Population-based linkage of health records in Western Australia: development of a health services research linked database. Aust N Z J Public Health 1999, 23:453-459.

9. Australian Bureau of Statistics: Australian Historical Population Statistics, 2006. Commonwealth of Australia, Canberra; 2006.

10. Stanley FJ, Croft ML, Gibbins J, Read AW: A population database for maternal and child health research in Western Australia using record linkage. Paediatr Perinat Epidemiol 1994, 8:433-447.

11. Read AW, Gibbins J, Stanley FJ, Morich P: Hospital admissions before the age of 2 years in Western Australia. Arch Dis Child 1994, 70:205-210.

12. Brameld KJ, Holman CD, Bass AJ, Codde JP, Rouse IL: Hospitalisation of the elderly during the last year of life: an application of record linkage in Western Australia 1985-1 994. J Epidemiol Community Health 1998, 52:740-744.

13. Williams K, Leonard H, Tursan d'Espaignet E, Colvin L, Slack-Smith L, Stanley F: Hospitalisations from birth to five years in a population cohort of Western Australian children with intellectual disability. Arch Dis Child 2005, 90:1243-I248.

14. Freemantle CJ, Read AW, de Klerk NH, McAullay D, Anderson IP, Stanley FJ: Patterns, trends, and increasing disparities in mortality for Aboriginal and non-Aboriginal infants born in Western Australia, 1980-200I: population database study. The Lancet 2006, 367: 1758-1766.

15. World Health Organisation: Manual of the international statistical classification of diseases, injuries and causes of death. International classification of diseases 9th revision edition. Geneva: World Health Organisation; 1977.

16. SAS/STAT: Copyright (c) 1999-200I by SAS Institute Inc. Cary, NC, USA.

17. Copeland GE, Kirby RS: Using birth defects registry data to evaluate infant and childhood mortality associated with birth defects: an alternative to traditional mortality assessment using underlying cause of death statistics. Birth Defects Res $A$ Clin Mol Teratol 2007, 79:792-797.

18. MacFaul R, Werneke U: Recent trends in hospital use by children in England. Arch Dis Child 2001, 85:203-207.

19. Leonard S, Bower C, Petterson B, Leonard H: Survival of infants born with Down's syndrome: 1980-96. Paediatr Perinat Epidemiol 2000, 14:163-171.

20. Slack-Smith L, Colvin L, Leonard H, Kilpatrick N, Bower C, Brearley Messer L: Factors associated with dental admissions for children under five years in Western Australia. Arch Dis Child 2008. Published Online First: 5 December 2008

2I. Hall JG, Powers EK, McLlvaine RT, Ean VH, Opitz JM: The frequency and financial burden of genetic disease in a pediatric hospital. Am J Med Genet 1978, I:417-436.

22. Carnevale A, Hernández M, Reyes R, Paz F, Sosa C, Opitz JM, Reynolds JF: The frequency and economic burden of genetic disease in a pediatric hospital in Mexico city. Am J Med Genet 1985, 20:665-675

23. Viner RM: National survey of use of hospital beds by adolescents aged 12 to 19 in the United Kingdom. BMJ 200I, 322:957-958.

24. Newacheck PW, Kim SE: A national profile of health care utilization and expenditures for children with special health care needs. Arch Pediatr Adolesc Med 2005, 159:10-17.

25. Waitzman NJ, Romano PS, Scheffler RM: Estimates of the economic costs of birth defects. Inquiry 1994, 31 : I 88-205.

26. Casselbrant ML, Mandel EM: Epidemiology. In Evidence-Based Otitis Media Edited by: Rosenfeld RM, Bluestone CD. London: B.C. Decker Inc; 1999:1 17-136.

27. Bluestone CD: Eustachian Tube Function and Dysfunction. In Evidence-Based Otitis Media Edited by: Rosenfeld RM, Bluestone CD. London: B.C. Decker Inc; 1999:137-149.

28. Centers for Disease Control and Prevention: Hospital stays, hospital charges, and in-hospital deaths among infants with selected birth defects - United States, 2003. MMWR Morb Mortal Wkly Rep 2007, 56:25-29.

\section{Pre-publication history}

The pre-publication history for this paper can be accessed here:

http://www.biomedcentral.com/1471-2431/9/32/prepub 